\title{
FARINHA DO MESOCARPO DO BABAÇU EM RAÇÕES DE FRANGOS DE CORTE NA FASE INICIAL
}

\author{
BABASSU MESOCARP FLOUR IN BROILER DIETS AT THE INITIAL STAGE
}

Cruz, L.A. ${ }^{1 *} ;$ Vaz, R.G.M.V. ${ }^{1 A}$; Siqueira, J.C. ${ }^{18} ;$ Silva, G.F. ${ }^{1 C} ;$ Rodrigues, K.F. ${ }^{1 D}$; Stringhini, J.H. ${ }^{1 \mathrm{E}}$; Ramos, A.T. ${ }^{\mathrm{IF}}$; De Sousa, J.P. ${ }^{1 \mathrm{G}}$; Calixto, M. ${ }^{1 \mathrm{H}}$ e Fonseca, F.L.R. ${ }^{11}$

${ }^{1}$ Departamento de Ciência Animal. UFT. Programa de Pós-graduação em Ciência Animal Tropical.

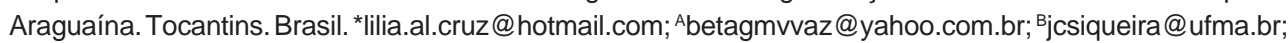
'Gerson@mail.uft.edu.br; ${ }^{\circ}$ rodrigueskf@mail.uft.edu.br; Ehenrique@vet.ufg.br Fadrianotony@hotmail.com; Gjp-zoot@hotmail.com; ${ }^{H}$ monicacalixto_@hotmail.com; 'flavialindy_182@hotmail.com

\section{Palavras chave adicionais}

Alimento alternativo. Co-produto. Proteínas plasmáticas.

\section{RESUMO}

Objetivou-se avaliar o desempenho produtivo, rendimento de carcaça e proteínas do sangue de 200 frangos de corte (machos, Cobb 500), no período de 8 a 21 dias de idade, alimentados com dietas a base de milho e farelo de soja contendo $0,6,12$ e $18 \%$ de farinha do mesocarpo de babaçu (Orbignya martiana) (FMB), distribuídos em delineamento experimental inteiramente casualizado com 4 tratamentos (níveis de FMB, rações formuladas para serem isoenergéticas e isoprotéicas), 5 repetições, sendo cada parcela experimental composta por 10 aves. Aos 21 dias de idade as aves foram abatidas para avaliação de rendimento de carcaça, e proteínas do sangue. A inclusão da FMB não afetou o consumo de ração, porém piorou o ganho de peso e a conversão alimentar no nível de $18 \%$. O rendimento de carcaça foi afetado nos maiores níveis de inclusão. Os menores níveis de proteína total e globulinas foram observados na inclusão de $6 \%$. Conclui-se que a adição de até $12 \%$ de farinha do mesocarpo de babaçu nas rações não compromete o desempenho. A inclusão de FMB aumenta o custo por $\mathrm{kg}$ de frango produzido e reduz a margem bruta, sendo economicamente inviável.

\section{SUMMARY}

The objective of this work was to evaluate the productive performance, carcass yield and blood

\section{Additional KeYWORdS}

Alternative food. Byproduct. Plasma proteins.

proteins of 200 male broilers (Cobb 500) on the 821 days of age period, fed diets based on corn and soybean meal containing $0,6,12$ and $18 \%$ of babassu (Orbignya martiana) mesocarp flour (FMB), distributed in a completely randomized design with four treatments (levels of FMB, diets formulated to be isocaloric and isonitrogenous), five replicates and a experimental unit with 10 broilers. At 21 days old the broilers were slaughtered to evaluate carcass yield, and blood proteins. The inclusion of FMB not affected feed intake, but decreased the weight gain and feed conversion in the level $18 \%$. Carcass yield was affected in the highest levels of inclusion. The levels of total protein and globulins were the lower in the $6 \%$ of inclusion. In conclusion, the addition up to $12 \%$ of FMB did not compromise the performance of broiler. The inclusion of this ingredient increases the cost per $\mathrm{kg}$ of chicken produced and decreases the gross margin, being economically unviable.

\section{INTRODUÇÃO}

O milho e farelo de soja, respondem por aproximadamente $90 \%$ do total da ração, contribuindo assim para o elevado custo relacionado à alimentação, e consequentemente para os custos totais de produção de frangos de corte (Pascoal et al., 2006). 
A preocupação pela busca de ingredientes alternativos é plenamente justificável (Jácome et al., 2002). Dentre eles, destacase o babaçu, que é nativo das regiões Norte, Nordeste e Centro Oeste do Brasil, mas também pode ser encontrado em outros países como Bolívia, México e Peru. No Brasil o babaçu ocupa de 13 a 18 milhões de ha, nos estados do Maranhão, Piauí, Tocantins, Goiás, Mato Grosso, Amazonas, Pará, Ceará, Bahia e Minas Gerais, perfazendo um total de 279 municípios (Brasil, 2009).

Do babaçu inúmeros produtos podem ser obtidos sendo os mais expressivos o carvão e o óleo. A, partir de um processo mecanizado, obtêm-se os resíduos, sendo que os originados do mesocarpo formam as farinhas, que recebem três classificações: farinha orgânica, farinha média e farinha fina, diferindo apenas pela textura e granulometria (Santos Neta, 2010).

De acordo com Pavlak et al. (2007), $10 \%$ do fruto é do mesocarpo fibroso e seu valor varia em torno de $52 \%$ de amido na composição do mesocarpo.

Objetivou-se com este trabalho avaliar diferentes níveis de inclusão de farinha do mesocarpo do babaçu (FMB) na dieta de frangos de corte de 8 a 21 dias de idade sobre as variáveis de desempenho (consumo de ração, ganho de peso, conversão alimentar), rendimento de carcaça e proteínas do sangue.

\section{MATERIALE MÉTODOS}

O experimento foi conduzido no setor de avicultura da Universidade Federal do Tocantins, Araguaína - TO, no período de 17 a 30 de março de 2011. Foi utilizado um galpão convencional disposto no sentido Leste-Oeste, coberto com palha de babaçu, com piso de concreto dotado de gaiolas metabólicas com área de $1 \mathrm{~m}^{2}$.

Foram utilizados 200 pintos machos da linhagem Cobb $500^{\circledR}$, alimentados com ração formulada para atender suas exigências
(Rostagno et al., 2005) até o sétimo dia de vida. No oitavo dia iniciou-se o experimento com animais apresentando peso médio de $145 \mathrm{~g}$. As aves foram pesadas individualmente para constituir parcelas com pesos iniciais homogêneos, distribuídos em gaiolas metabólicas $(1,0$ x 1,0 x 0,5 m) em um delineamento experimental inteiramente casualizado com quatro tratamentos e cinco repetições, sendo cada unidade experimental composta por 10 aves.

As gaiolas continham um sistema de aquecimento com lâmpadas de 60 watts, comedouro e bebedouro tipo calha. A limpeza dos bebedouros e o fornecimento de água e ração foram realizados duas vezes ao dia, possibilitando a ingestão de água e o consumo de ração ad libitum. O programa de luz adotado foi de 24 horas luz (natural + artificial).

As condições ambientais no interior das instalações foram registradas diariamente seis vezes ao dia (08:00, 10:00, 12:00, 14:00, 16:00 e 18:00 h), por meio de termômetros de máxima e mínima, bulbo seco, bulbo úmido e globo negro, dos quais foram convertidos em ITGU (índice de temperatura globo e umidade), de acordo com Buffington et al. (1981) para caracterização ambiental, pela equação:

$I T G U=\operatorname{Tgn}+0,36 T p o-330,08$

onde:

Tgn= temperatura de globo negro $(K)$;

$\mathrm{Tpo}=$ temperatura de ponto de orvalho $(\mathrm{K})$.

Os tratamentos consistiram de uma ração basal a base de milho e farelo de soja ( $0 \%$ de inclusão de farinha do mesocarpo de babaçu (Orbignya martiana) e outros três tratamentos com níveis crescentes de FMB $(6,0 ; 12,0$ e $18,0 \%)$. As rações experimentais foram formuladas considerando a composição química dos ingredientes (tabela I), para conterem $3000 \mathrm{kcal} / \mathrm{kg}$ de energia metabolizável e $20 \%$ de proteína bruta, de acordo com Rostagno et al. (2005) (tabela II). 


\section{FARINHA DO MESOCARPO DO BABAÇU EM RAÇÕES DE FRANGOS DE CORTE}

As variáveis de desempenho avaliadas foram consumo de ração (CR), ganho de peso (GP), e conversão alimentar (CA). O consumo de ração foi calculado pela diferença entre o fornecido e as sobras, sendo o ganho de peso calculado pela diferença entre o peso das aves no início e no final do experimento. A partir dos dados de consumo de ração e ganho de peso calculou-se a conversão alimentar (CR/GP).

No $21^{\circ}$ dia de vida todas as aves foram pesadas, e após um período de 12 horas de jejum, foram selecionadas duas aves com peso próximo à média $( \pm 5 \mathrm{~g})$ da parcela para avaliar o rendimento de carcaça (com cabeça, pescoço e pés): peso da carcaça depenada e eviscerada/peso vivo em jejum expresso em porcentagem. As aves foram abatidas por deslocamento cervical, san-

Tabela I . Composição dos ingredientes utilizados na formulação das rações (com base na matéria natural). (Composition of the ingredients used in formulating the diets (based on fresh matter)).

\begin{tabular}{lccc}
\hline & Milho & $\mathrm{FS}^{1}$ & $\mathrm{FMB}^{*}$ \\
\hline Energia bruta (kcal/kg) & 3381 & 2256 & 3,689 \\
Proteína bruta (\%) & 8,28 & 45,32 & 3,06 \\
Cálcio (\%) & 0,030 & 0,240 & 0,10 \\
Fósforo disponível (\%) & 0,080 & 0,180 & $\mathrm{ND}$ \\
Cloro (\%) & 0,050 & 0,050 & $\mathrm{ND}$ \\
Potássio (\%) & 0,280 & 1,830 & $\mathrm{ND}$ \\
Sódio (\%) & 0,020 & 0,020 & $\mathrm{ND}$ \\
Lisina total (\%) & 0,240 & 2,770 & $\mathrm{ND}$ \\
Metionina total (\%) & 0,170 & 0,640 & $\mathrm{ND}$ \\
Met+cistina total (\%) & 0,360 & 1,370 & $\mathrm{ND}$ \\
Treonina total (\%) & 0,320 & 1,780 & $\mathrm{ND}$ \\
Triptofano total (\%) & 0,070 & 0,620 & $\mathrm{ND}$ \\
Fibra bruta (\%) & 2,60 & 5,30 & 37,33 \\
Extrato etéreo (\%) & 3,61 & 1,66 & $\mathrm{ND}$ \\
\hline
\end{tabular}

${ }^{1}$ Rostagno et al. (2005).

$\mathrm{FS}=$ farelo de soja; $\mathrm{FMB}$ = farinha do mesocarpo do babaçu; $N D=$ não definido.

*Laboratório de Nutrição Animal. Centro de Ciência Animal Tropical. Universidade Federal do Tocantins. gradas, escaldadas, depenadas e evisceradas.

No momento da sangria foi coletado o sangue, sendo encaminhado para o laboratório de Patologia Clínica da Universidade

Tabela II. Composição das rações contendo farinha do mesocarpo do babaçu (FMB) para frangos de 8 a 21 dias de idade. (Composition of diets contaning babassu mesocarp flour (FMB) for broilers at 8 to 21 days old).

\begin{tabular}{lcccc}
\hline & \multicolumn{4}{c}{ Níveis de FMB (\%) } \\
& 0 & 6 & 12 & 18 \\
\hline Milho & 62,39 & 53,58 & 44,77 & 36,81 \\
FS (45\%) & 32,97 & 34,25 & 35,53 & 35,95 \\
FMB & 0,00 & 6,00 & 12,00 & 18,00 \\
Fb & 1,78 & 1,80 & 1,83 & 1,85 \\
Óleo de soja & 1,04 & 2,58 & 4,13 & 5,67 \\
Calcário & 0,77 & 0,72 & 0,67 & 0,62 \\
Sal comum & 0,43 & 0,43 & 0,44 & 0,44 \\
DL-Met & 0,24 & 0,26 & 0,28 & 0,29 \\
L-Lisina HCl & 0,24 & 0,22 & 0,20 & 0,19 \\
L-Treonina & 0,06 & 0,07 & 0,07 & 0,08 \\
Núcleo' & 0,04 & 0,04 & 0,04 & 0,04 \\
Total & 100 & 100 & 100 & 100 \\
EM (kcal/kg) & 3000 & 3000 & 3000 & 3000 \\
PB (\%) & 20,79 & 20,79 & 20,79 & 20,79 \\
Cálcio (\%) & 0,88 & 0,88 & 0,88 & 0,88 \\
P disponível (\%) & 0,44 & 0,44 & 0,44 & 0,44 \\
P total (\%) & 0,71 & 0,71 & 0,70 & 0,70 \\
Lisina total (\%) & 1,26 & 1,26 & 1,26 & 1,26 \\
Met+cys total (\%) & 0,89 & 0,89 & 0,89 & 0,89 \\
Met total (\%) & 0,56 & 0,57 & 0,58 & 0,59 \\
Sódio (\%) & 0,21 & 0,21 & 0,21 & 0,21 \\
Treonina total (\%) & 0,85 & 0,85 & 0,85 & 0,85 \\
FB (\%) & 3,37 & 5,45 & 7,53 & 9,58 \\
EE (\%) & 3,84 & 5,07 & 6,32 & 7,57 \\
\hline
\end{tabular}

$\mathrm{FS}=$ farelo de soja; $\mathrm{Fb}=$ fosfato bicálcico; $\mathrm{PB}=$ proteína bruta; $\mathrm{FB}=$ fibra bruta; $\mathrm{EE}=$ extrato etéreo. ${ }^{1}$ Composição/kg: Mn, 18,175 mg; Zn, 17,5 mg; Fe, 11,25; Cu, 2 g; I, 187,5 mg; Se, 75 mg; vitamina K3, $360 \mathrm{mg}$; vitamina B1, 436,5 mg; vitamina B2, 4300 $\mathrm{mg}$; vitamina B6, $624 \mathrm{mg}$; vitamina B12, $2400 \mathrm{mg}$; ácido fólico, $200 \mathrm{mg}$; ácido pantotênico, 3,12 mg; niacina, $8,40 \mathrm{mg}$; biotina, $10,0 \mathrm{mg}$; colina, 78,102 $\mathrm{mg}$; aditivo antioxidante, $25,0 \mathrm{mg}$; halquinol, 7500 $\mathrm{mg}$; nicarbazina, $27,5 \mathrm{mg}$. 


\section{CRUZ ET AL.}

Federal do Tocantins, onde foi centrifugado a $3000 \mathrm{rpm}$ durante 15 minutos para a obtenção do plasma, que foi armazenado a $-20^{\circ} \mathrm{C}$. Foram avaliadas as proteínas totais (albumina e globulinas) sendo esta última obtida pela diferença entre a concentração das proteínas totais e a albumina, com o auxilio de técnicas foto colorimétricas utilizando kits reagentes (Labtest Diagnóstica, Brasil).

Todas as variáveis de desempenho, rendimento de carcaça e proteínas sanguíneas foram submetidos aos testes de normalidade (Cramer Von Mises) e Homocedasticidade (Levene). Satisfeitas estas pressuposições foram submetidas à análise de variância segundo o modelo estatístico:

$$
Y_{i j}=\mu+F M B_{i}+e_{i j}
$$

onde:

$\mathrm{Y}_{\mathrm{ij}}=$ valor observado para a variável dependente no i-ésimo nível de inclusão da FMB; j-ésima repetição:

$\mu=$ efeito da média geral;

$\mathrm{FMB}_{\mathrm{i}}=$ efeito do i-ésimo nível de inclusão da FMB na ração;

$e_{i j}=$ erro experimental.

Nas variáveis cujos efeitos da inclusão da FMB foram detectados pela análise de variância, realizaram-se análises de regressão por meio de modelos polinomiais. As médias de cada tratamento também foram comparadas por meio do teste SNK. As análises estatísticas foram realizadas com o auxílio do software SAS 9.0, por meio do procedimento GLM.

Determinou-se o custo com alimentação por kg de frango produzido como segue ${ }^{1}$ :

$\mathrm{R} \$ / \mathrm{kgGP} \mathrm{P}_{\mathrm{i}}=\left(\mathrm{CR}_{\mathrm{i}} \times \mathrm{R} \$ \mathrm{R}_{\mathrm{i}}\right) / \mathrm{GP}_{\mathrm{i}}$;

com $\mathrm{i}=1,2,3,4$.

Emque:

$\mathrm{R} \$ / \mathrm{kgGP}=$ custo por $\mathrm{kg}$ de frango $(\mathrm{R} \$ / \mathrm{kg})$ produzido com a utilização do i-ésimo nível de inclusão da

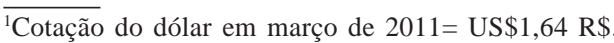

farinha do mesocarpo do babaçu;

$\mathrm{CR}_{\mathrm{i}}=$ quantidade de ração $(\mathrm{kg})$ consumida no iésimo nível de inclusão da farinha do mesocarpo do babaçu;

$\mathrm{R} \$ \mathrm{R}_{\mathrm{i}}=$ custo da ração $(\mathrm{R} \$ / \mathrm{kg})$ contendo o i-ésimo nível de inclusão da farinha do mesocarpo do babaçu;

$\mathrm{GP}_{\mathrm{i}}=$ ganho de peso $(\mathrm{kg})$ das aves que receberam o i-ésimo nível inclusão da farinha do mesocarpo do babaçu.

A margem por kg de frango para cada nível de inclusão da farinha do mesocarpo de babaçu nas rações foi calculada pela expressão:

$$
M_{i}=P V F-R \$ / k g G P_{i}
$$

em que:

$M_{i}=$ margem por $\mathrm{kg}$ de frango $(R \$)$ obtida com a utilização do i-ésimo nível de inclusão da farinha do mesocarpo do babaçu;

$P V F=$ preço de venda do frango vivo $(R \$ / k g)$;

$\mathrm{R} \$ / \mathrm{kgGP}=$ custo por $\mathrm{kg}$ de frango $(\mathrm{R} \$ / \mathrm{kg})$ produzido com a utilização do i-ésimo nível de inclusão da farinha do mesocarpo do babaçu.

\section{RESULTADOSEDISCUSSÃO}

Os dados de climatologia estão apresentados na tabela III.

O valor de temperatura média de globo negro no interior do galpão foi de $30,85^{\circ} \mathrm{C}$, e a umidade relativa média foi de 55,0\%, satisfatória para frangos de corte entre $50 \mathrm{e}$ 70 \% segundo Tinôco (2001) e Medeiros et al. (2005). A amplitude térmica foi de 25,3 a $30,6^{\circ} \mathrm{C}$, sendo o ITGU de 77,8. Para Medeiros

Tabela III. Caracteristicas ambientais durante o período experimental. (Environmental characteristics during the experimental period).

\begin{tabular}{lc}
\hline & Média \pm desvio padrão \\
\hline Globo negro $\left({ }^{\circ} \mathrm{C}\right)$ & $30,8 \pm 2,14$ \\
T máxima $\left({ }^{\circ} \mathrm{C}\right)$ & $30,6 \pm 1,70$ \\
T mínima $\left({ }^{\circ} \mathrm{C}\right)$ & $25,3 \pm 1,65$ \\
UR $(\%)$ & $55,0 \pm 5,5$ \\
ITGU & $77,8 \pm 1,4$ \\
\hline
\end{tabular}


Tabela IV. Desempenho de frangos de corte de 8 a 21 dias de idade, de acordo com o nível de inclusão de farinha do mesocarpo do babaçu. (Performance of 8-21 days old broilers according to level of babassu mesocarp flour).

\begin{tabular}{lcccccc}
\hline \multicolumn{7}{c}{ Níveis de farinha do mesocarpo de babaçu } \\
& $0 \%$ & $6 \%$ & $12 \%$ & $18 \%$ & p>F & CV \\
\hline Consumo de ração (g) & $803,8 \pm 21,0$ & $834,0 \pm 41,8$ & $797,3 \pm 27,9$ & $833,2 \pm 38,2$ & 0,2086 & 3,88 \\
Ganho de peso (g) & $529,2^{\mathrm{a}} \pm 8,5$ & $534,4^{\mathrm{a}} \pm 3,8$ & $513,2^{\mathrm{a}} \pm 16,4$ & $479,2^{\mathrm{b}} \pm 12,2$ & 0,0004 & 3,25 \\
Conversão alimentar (g/g) & $1,52^{\mathrm{a}} \pm 0,03$ & $1,56^{\mathrm{a}} \pm 0,07$ & $1,55^{\mathrm{a}} \pm 0,07$ & $1,74^{\mathrm{b}} \pm 0,07$ & 0,0002 & 3,93 \\
Rendimento de carcaça (\%) & $77,7^{\mathrm{a}} \pm 0,72$ & $79,0^{\mathrm{a}} \pm 0,60$ & $76,8^{\mathrm{b}} \pm 1,1$ & $76,6^{\mathrm{b}} \pm 1,4$ & 0,0198 & 1,38 \\
\hline
\end{tabular}

$\mathrm{F}=$ teste $\mathrm{F}$ da análise de variância; $\mathrm{CV}=$ coeficiente de variação (\%).

a,b Médias seguidas de letras iguais não diferem pelo teste SNK $(p>0,05)$.

et al. (2005), um ITGU para ambientes confortáveis está na faixa de 69 a 77, sendo que em uma escala de 78 a 88 são considerados ambientes quentes.

Os resultados de desempenho obtidos na fase inicial evidenciaram que os níveis de inclusão da FMB não influenciaram o consumo de ração, porém, houve uma diminuição no ganho de peso e uma piora na conversão alimentar das aves $(\mathrm{p}<0,05)$ com o nível de $18 \%$ de inclusão (tabela IV). As equações ajustadas foram:
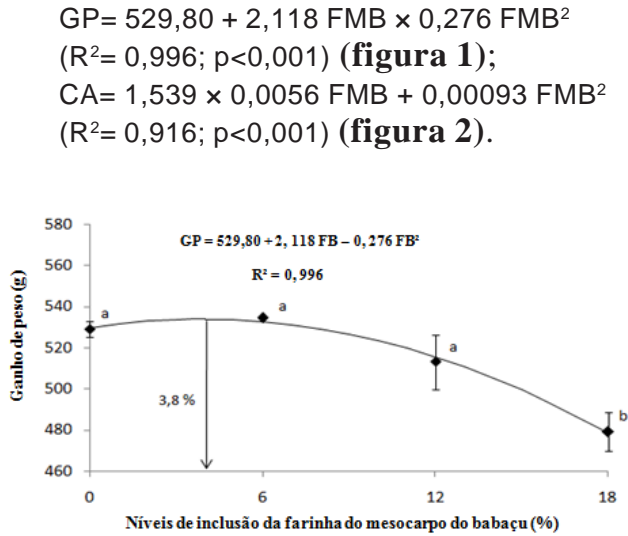

Figura 1. Niveis de farinha do mesocarpo de babaçu sobre o ganho de peso de frangos de corte na fase de 8 a 21 dias de idade. (Effect of levels of babassu mesocarp flour on the weight gain during 8-21 days of age).
Como ilustra a tabela I, os teores de fibra bruta da FMB são elevados, o que contribui para aumentar a taxa de passagem do alimento no trato gastrointestinal, consequentemente o aproveitamento dos nutrientes são reduzidos (Castro Junior et al., 2005). Este fato ajuda explicar a queda de desempenho com a utilização do nível de $18 \%$ de inclusão da FMB.

Com base no teste SNK, não houve diferença até a inclusão de $12 \%$, para estas variáveis, o que abona a utilização deste nível nas rações. De acordo com Sampaio (2007) a natureza quantitativa dos trata-

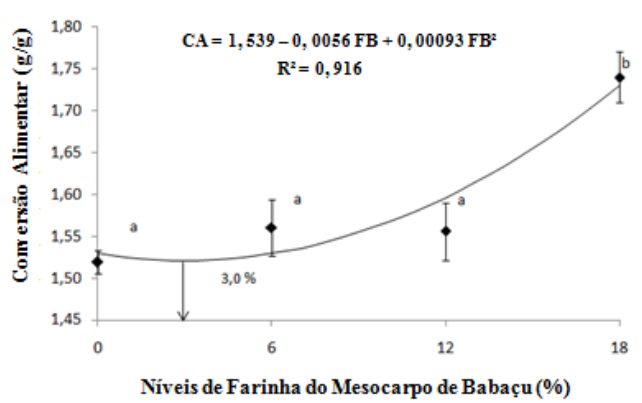

Figura 2. Niveis de farinha do mesocarpo de babaçu sobre a conversão alimentar de frangos de corte na fase de 8 a 21 dias de idade. (Effect of levels of babassu mesocarp flour on the feed conversion rate of broilers during 8-21 days of age). 
mentos empregados sugere o estudo de modelos, porém este procedimento por si só, não substitui a comparação de médias.

$\mathrm{O}$ rendimento de carcaça dos frangos aos 21 dias de idade reduziu de maneira linear com os níveis de inclusão da FMB, segundo a equação:

$$
\begin{aligned}
& R C=78,2469-0,08492 \text { FB } \\
& \left(R^{2}=0,4179, p<0,001\right) \text { (figura 3). }
\end{aligned}
$$

Esse decréscimo pode ser explicado, em parte, pela diminuição do GP no nível de $18 \%$, o que contribui para menor de rendimento de carcaça. Entretanto, pelo teste de SNK, foi possível observar que até o nível de $6 \%$ de inclusão da FMB nas rações não houve comprometimento do RC (tabela IV).

Considerando os parâmetros bioquímicos do sangue aos 21 dias de idade, observou-se diferença significativa para os níveis de proteína total $(\mathrm{p}<0,05)$, sendo que os níveis de 0,12 e $18 \%$, apresentaram concentrações mais elevadas (tabela $\mathbf{V}$ ), sendo a equação:

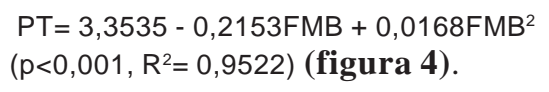

O mesmo comportamento foi observado

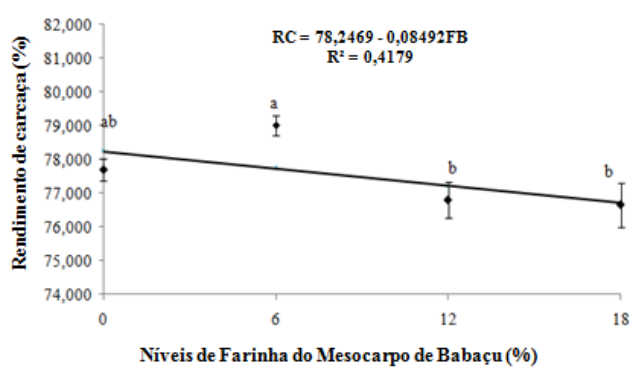

Figura 3. Niveis de farinha do mesocarpo de babaçu sobre o rendimento de carcaça na fase de 8 a 21 dias de idade. (Effect of levels of babassu mesocarp flour on the carcass yield during 8-21 days of age).

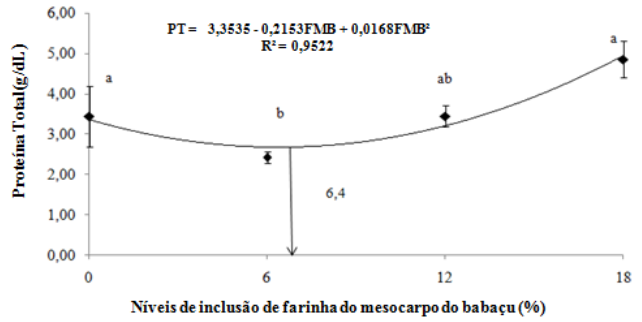

Figura 4. Niveis de farinha do mesocarpo de babaç sobre as proteínas plasmáticas totais $(\mathrm{g} / \mathrm{dL})$ do sangue de frangos de corte na fase de 8 a 21 dias de idade. (Effect of levels of babassu mesocarp flour on total blood plasma proteins of broilers during 8-21 days of age).

para os níveis de globulina obtendo-se a equação:

$\mathrm{GLOB}=1,3281-0,1973 \mathrm{FMB}+0,0144 \mathrm{FMB}^{2}$
$\left(\mathrm{p}<0,001, \mathrm{R}^{2}=0,9857\right)($ figura 5).

Para os níveis de albumina não foram observadas diferença significativa $(p>0,05)$. Os pontos de mínimo foram 6,4 e 6,8 \% para proteínas totais e globulinas, respectivamente, apontando esses níveis de inclusão como os mais satisfatórios para essas variáveis.

Não há trabalhos que avaliaram as proteínas plasmáticas de frangos de corte submetidos à inclusão de FMB, mas o

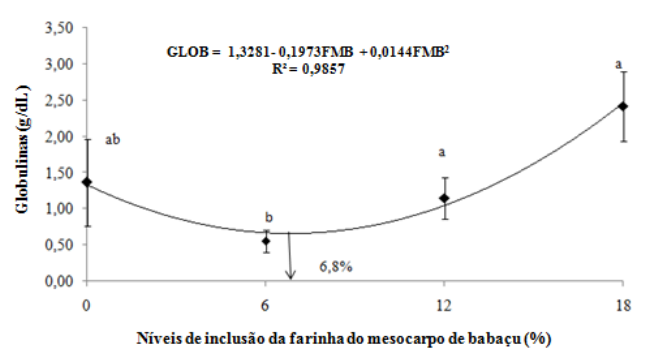

Figura 5. Níveis de farinha do mesocarpo de babaçu sobre as globulinas ( $\mathrm{g} / \mathrm{dL}$ ) de frangos de corte na fase de 8 a 21 dias de idade. (Effect of levels of babassu mesocarp flour on the globulins of broilers during 8-21 days of age). 
Tabela $\boldsymbol{V}$. Proteínas do soro de frangos de corte aos 21 dias de idade. (Serum proteins in broilers at 21 days old).

\begin{tabular}{lcccccc}
\hline \multicolumn{7}{c}{ Níveis de farinha do mesocarpo de babaçu } \\
$0 \%$
\end{tabular}

$\mathrm{CV}=$ Coeficiente de variação (\%); $\mathrm{F}=$ teste $\mathrm{F}$ da análise de variância.

a,bMédias seguidas de letras iguais não diferem pelo teste SNK $(p>0,05)$.

comportamento apresentado para essa variável pode ter sido em virtude da inclusão da FMB, bem como de fatores como idade, sazonalidade e condições de manejo, fatores que podem alterar os parâmetros bioquímicos do sangue (Lumiej, 1990).

No presente estudo observou-se que as globulinas apesar de apresentarem diferenças, permaneceram abaixo dos níveis normais em todos os tratamentos $(3,1 \mathrm{~g} / \mathrm{dL})$ e as proteínas totais permaneceram próximo ao valor referência de 5,6 g/dL. De acordo com Campbell e Dein (1984), a concentração de proteína sérica total nas aves comparadas a mamíferos é menor variando em torno de 3,0 e 6,0 g/dL.

Com base nos preços dos insumos utilizados na fabricação das rações experimentais, observou-se que a inclusão de níveis crescentes da FMB promoveu aumento linear no preço das rações (tabela VI), consequência, principalmente, do aumento dos níveis de óleo de soja à medida que os níveis de FMB aumentaram (tabela II). O menor custo com ração por kg de GP foi obtido com a ração basal ( $0 \%$ de inclusão da FMB), o que resultou na maior margem bruta (tabela VI).

\section{CONCLUSÃO}

Conclui-se que a adição de até $12 \%$ de farinha do mesocarpo de babaçu nas rações não compromete o desempenho de frangos de corte de 8 a 21 dias de idade, entretanto, a inclusão deste ingrediente aumenta o custo por $\mathrm{kg}$ de frango produzido e reduz a margem bruta, sendo economicamente inviável.

Tabela VI. Dados econômicos obtidos com frangos de corte de 8 a 21 dias de idade recebendo farinha de babaçu. (Economic data obtained with broilers from 8 to 21 days feed with babassu flour).

\begin{tabular}{|c|c|c|c|c|}
\hline & \multicolumn{4}{|c|}{ Níveis de farinha do mesocarpo de babaçu } \\
\hline & $0 \%$ & $6 \%$ & $12 \%$ & $18 \%$ \\
\hline Custo da ração ${ }^{1}(\mathrm{R} \$ / \mathrm{kg})$ & 0,931 & 0,949 & 0,967 & 0,979 \\
\hline Custo com ração por kg de GP (R $\$ / \mathrm{kg})$ & 1,141 & 1,481 & 1,503 & 1,702 \\
\hline Margem bruta ${ }^{2}(\mathrm{R} \$ / \mathrm{kg})$ & 1,436 & 1,369 & 1,347 & 1,148 \\
\hline
\end{tabular}

${ }^{1}$ Considerando os seguintes preços: milho $=R \$ 0,59 / \mathrm{kg}$; farelo de soja $=R \$ 1,30 / \mathrm{kg}$; farinha do mesocarpo de babaçu= $R \$ 0,22 / \mathrm{kg}$; fosfato bicálcico $=R \$ 2,77 / \mathrm{kg}$; Óleo de soja $=R \$ 2,49 / \mathrm{kg}$; calcário $=R \$ 0,43 / \mathrm{kg}$; sal= 0,80/kg; DL-metionina $=R \$ 11,87 / \mathrm{kg} ; \mathrm{L}$-lisina $\mathrm{HCl}=\mathrm{R} \$ 6,67 / \mathrm{kg} ;$ L-treonina $=\mathrm{R} \$ 6,76 / \mathrm{kg} ;$ suplemento mineral e vitamínico $=R \$ 9,00 / \mathrm{kg}$. ${ }^{2}$ Considerando o preço do frango vivo de $R \$ 2,85 / \mathrm{kg}$. 


\section{CRUZ ET AL.}

\section{BIBLIOGRAFIA}

Brasil. 2009. Promoção nacional da cadeia de valor do coco babaçu. Ministério do Meio Ambiente. http://www.territoriosdacidadania. gov.br/o/3738771 (10/02/2011).

Buffington, D.E.; Colazzo-Arocho, A.; Canton, G.H.; Pitt, D.; Thatcher, W.W. and Wilcox, R.J. 1981. Black globe-humidity index (BGHI) as comfort equation for dairy cows. Transaction of the ASAE, 24: 711-714.

Castro Junior, F.G.; Camargo, J.C.M.; Gomes de Castro, A.M.M. e Budino, F.E.L. 2005. Fibra na alimentação de suínos. B Indust Anim, 62: 265280.

Campbell, T.W. and Dein, F.J. 1984. Avian hematology. The basics. Veterinary clinics of North America. J Small Anim Pract, 14: 223-48.

Jácome, I.M.T.D.; Silva, L.P.G.; Guim, A.; Lima, D.Q.; Almeida, M.M.; Araújo, M.J.; Oliveira, V.P.; Silva, J.D.B. e Martins, T.D.D. 2002. Efeitos da inclusão do farelo de coco nas rações de frangos de corte sobre o desempenho e rendimento da carcaça. Acta Sci Anim Sci, 24: 1015-1019.

Lumiej, J. 1990. Relation of plasma calcium to total protein and albumin in African grey (Psittacus erithacus) and amazon (Amazona spp.) parrots'. Avian Pathol, 19: 661-667.

Medeiros, C.M.;Baêta, F.C.; Oliveira, R.F.M.;Tinôco, I.F.F.; Albino, L.F.T.e Cecon, P.R. 2005. Efeitos da temperatura, umidade relativa e velocidade do ar em frangos de corte. Eng Agric, 13: 277286.

Pascoal, L.A.F.; Bezerra, A.P.A. e Gonçales, J.S.
2006. Farelo de babaçu: valor nutritivo e utilização na alimentação animal. Rev Eletrônica Nutritime, 3: 339-345.

Pavlak, M.C.M.;Zuniga, A.D.; Lima, T.L.A.; ArévaloPinedo, A.; Carreiro, S.C.; Fleury, C.S. e Silva, D.L. 2007. Aproveitamento da farinha do mesocarpo do babaçu (Orbignya martiana) para obtenção de etanol. Evidência, 7: 7-24.

Rostagno, H.S.; Albino, L.F.T.; Donzele, J.L.; Gomes, P.C.; Oliveira, R.F.; Lopes, D.C.; Ferreira, A.S. e Barreto, S.L. de T. 2005. Tabelas brasileiras para aves e suínos composição de alimentos e exigências nutricionais. UFV. Imprensa Universitária. Viçosa. 186 pp.

Rostagno, H.S.; Albino, L.F.T.; Donzele, J.L.; Gomes, P.C.; Oliveira, R.F.; Lopes, D.C.; Ferreira, A.S. e Barreto, S.L. de T. 2005. Tabelas brasileiras para aves e suínos composição de alimentos e exigências nutricionais. UFV. Imprensa Universitária. Viçosa. 186 pp.

Sampaio, I.B.M. 2007. Estatística aplicada à experimentação animal. Vol. 1. 3.a ed. FEPMVZ. Belo Horizonte. 264 pp.

Santos Neta, E.R. 2010. Avaliação de subprodutos do babaçu (Palmae: Orbignya martiana) na alimentação de frangos de corte. Dissertação (Mestrado em Ciência Animal Tropical). Escola de Medicina Veterinária e Zootecnia. Universidade Federal do Tocantins. Araguaína. 59 pp.

Tinôco, I.F.F. 2001. Avicultura industrial: novos conceitos de materiais, concepções e técnicas construtivas disponíveis para galpões avícolas brasileiros. Rev Bras Ciênc Avíc, 3: 1-26. 